\title{
Engineering Targeted Project Design in Kinematics and Control Classes
}

\author{
Debao Zhou \\ Department of Mechanical and Industrial Engineering \\ University of Minnesota, Duluth, MN 55812, USA \\ Email: dzhou@d.umn.edu
}

\begin{abstract}
Labs and projects will be the important ways to solidify the knowledge learned from classes since hands-on work can help the students understand class materials better. A kinematics and control lab class has been offered in the Department of Mechanical and Industrial Engineering here at the University of Minnesota, Duluth. The projects in class have been designed to solve actual engineering problems. Through the projects, the students will not only use the learned knowledge to build creative products, but also the student can understand class materials better and accumulate more interest in class knowledge. By building up this positive feedback, the learning achievements can be maximized.
\end{abstract}

\section{Introduction}

Kinematics and control classes focus on the design and control of mechatronics systems with multiple degrees of freedom. In kinematics class, both the design and analysis of planar and spatial linkages have been emphasized. The class is mainly concentrated on the study of the posture, velocity and acceleration of complex linkages. The System Dynamics and Control class provides the method to describe the dynamics of a mechanical system and the method to control its behaviors. Being offered labs on kinematics and control, the students can have the actual opportunity to use the knowledge on an actual system. However, since all the labs are predesigned and the students just followed the lab procedure, they still did not fully understand how to apply the knowledge to actual mechanical system. This is found out via the communication with the students. In order to make the students able to apply what they have learned from class, a project is designed during the kinematics and control labs. Through solving the problems they find in their everyday life, the students know exactly where the problems are and what they have to come out to solve them. After they have applied the knowledge and found how useful the knowledge is, they are excited about what they have done and build more interest on the class learning.

\section{Project Design Requirements}

For the project, the students are just asked to design, analyze and demonstrate a mechatronics system and provide evaluation to their final product, such as the analysis of the velocity, acceleration and position trajectory of a kinematic system and/or the analysis of the performance of their control system. The students are suggested to finish their design in three weeks by using totally 6 hours (two hours in each week). One group is formed by two students for discussion. In the first week, they are asked to do an extensive literature survey and find out what they want to build. In the second week, the students need to have a clear sketch about what they want to build and have the necessary components ordered. In the final week, the students are required to 
assemble their design and demonstrate how the design works. The students are also asked to prepare a comprehensive project report which includes introduction, literature survey, design description, results and discussion and conclusion. Finally, the students will be asked to give a 10 minutes presentation to introduce their design to the whole class.

Some equipment has been provided to the students. The first set of equipment is a kinematics kit [1] as shown in Figure 1. This kit includes a stepper motor, number of different links. The motor with control circuit is fixed on the white panel as shown in Figure 1. On the white panel distributed tapped holes with equal distance. The second set of equipment is a DC brush motor system with related data acquisition card, amplifier, power supply, function generator, oscilloscope and a computer with Matlab/Simulink as shown in Figure 2. The students are provided with the flexibility to disassemble the two sets of equipment to build their own design.

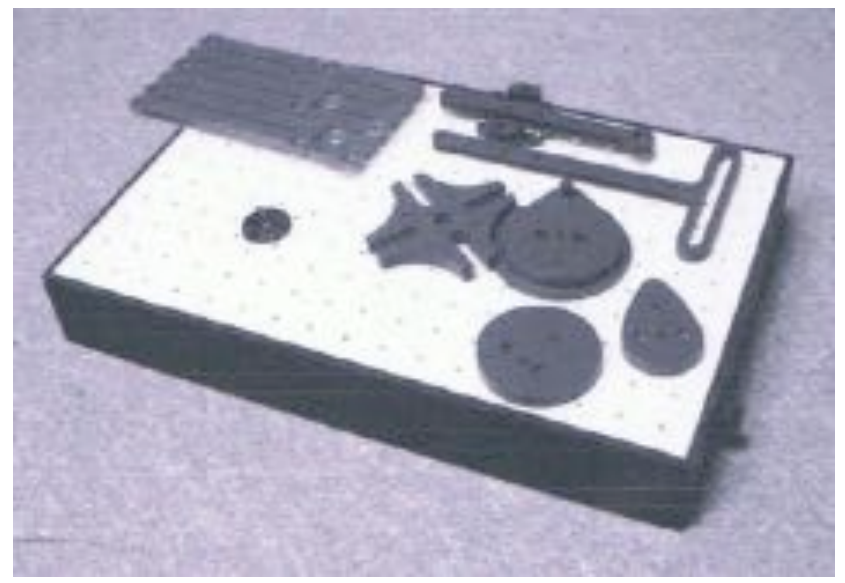

Figure 1, Kinematics kit (stepper motor is inside the box.

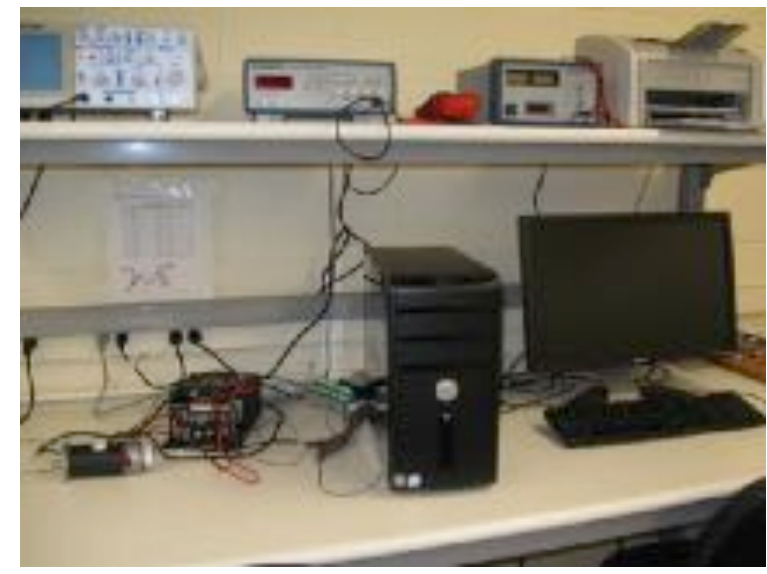

Figure 2, Motor and related equipment including hardware/software.

\section{Typical Projects}

In order to make the students finish the project in only 6 hours, the instructor suggested the students should consider one degree of freedom system in first priority such that the mechanism can be directly driven by the provided motors. Number of joints and links can be involved to achieve irregular or desired motion. The instructor also required them to make their design meaningful to fulfill certain engineering jobs. Due to this loss requirement and the connection with engineering, the instructor can feel that the students have great interest on the project design. They built a lot of interesting projects. Some of the projects are illustrated below.

\subsection{Control Concepts: Projects Involving Direct Motor Control}

Although you may think that a mechanism with one degree of freedom is simple, since it can be directly controlled by a motor, the students can verify the control theory in much easier way and they can concentrate on the understanding of the control theory. To realize the goal of real application, some students came out some wonderful ideas to apply the class materials, such as the ball balance project, light following for solar charger project, watering from a cup project, elevator simulator project, etc. 
The design of the ball balance project is shown in Figure 3, where the orientation of a long beam is directly controlled by a motor and the motor is control by a PID controller. A Sharp GP2Y0A21YK wide distance sensing optoelectronic sensor is used to feedback the ping-pong ball position. The project used a Zeigler Nichol's PID tuning method to get the best ball balancing results. This is a wonderful example to apply class learned technology to solve engineering problems.

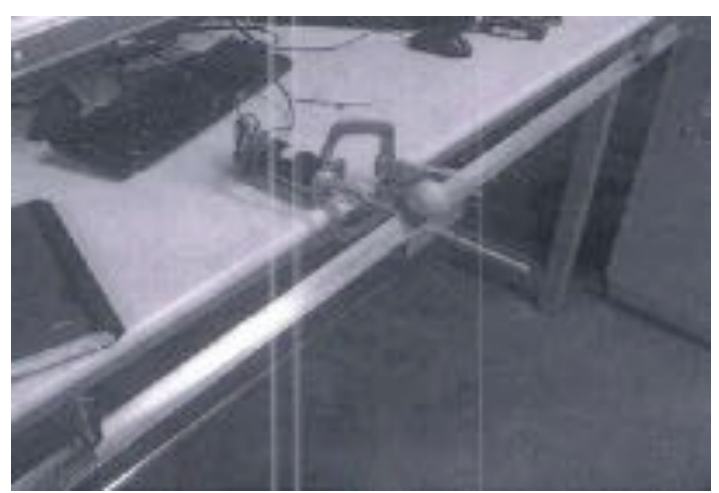

Figure 3, Ball balancing project.

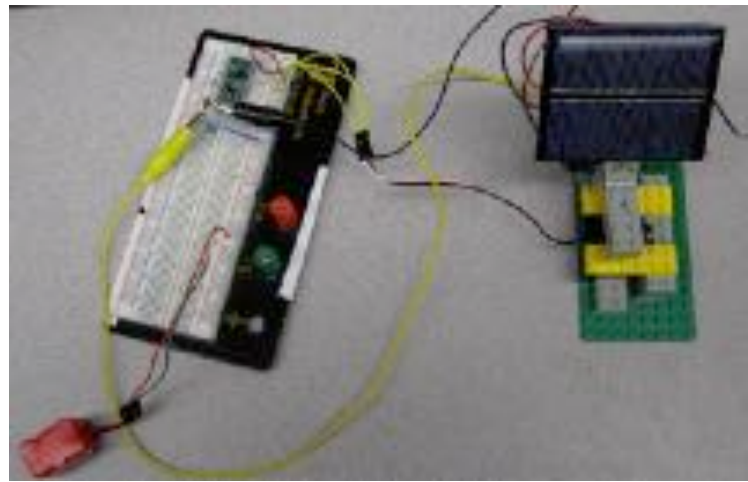

Figure 4, Light tracking for charging cell phone.

Another group of students used the direct control method to realize the tracking of sun light such that a cellular phone can be charged through a solar panel. The system is shown in Figure 4. A stepper motor was controlled by a microcontroller. PID controller is used in the microcontroller. The controller data are downloaded to the microcontroller from a personal computer after the tuning on computer. The feedback is from a light sensor which is just beneath the solar penal as shown in Figure 4.

After taking System Dynamics and Control class, the students have learned the basis of the classic control theories. However, they do not know how to apply them. By designing and controlling a mechanism themselves, the students have the strong feeling about where the controller is and how the controller works. This will build strong memory that they will never forget. Tuning in actual projects is the best way to gain and understand PID control theory.

\subsection{Kinematics Concepts: Projects Involving Crank-Slider Mechanism}

Complex planar linkages are involved in Kinematics class, where the analysis on position, velocity and acceleration may not very appealing to them. But when complex trajectory is involved, these planar linkages are very useful. Some of the students decided to realize some planar linkages and assign them actual applications. Two of the examples, the saw mechanism and the loco linkage, are shown below.

In the saw project, the students' though is to automate the wood sawing processing for labor saving. The slide crank mechanism learned from kinematics class will be an ideal candidate for this application. But the students did not stop after building this mechanism. They also did the kinematics analysis for the system. Through this experience, they know how to apply the learned knowledge to a real problem. Another group of students apply an accelerometer (ADXL321EB Dual-Axis Accelerometer from Analog Devices Inc. [3]) on the tip of the slider to measure the actual acceleration. Then the group compared the obtained acceleration with the calculated 
values using analytical method and the simulated acceleration from Solidworks Motion analysis software. After the comparison, what the students could say is "Amazing". Through such a project, they have much better feeling about the theoretical linkage analysis.

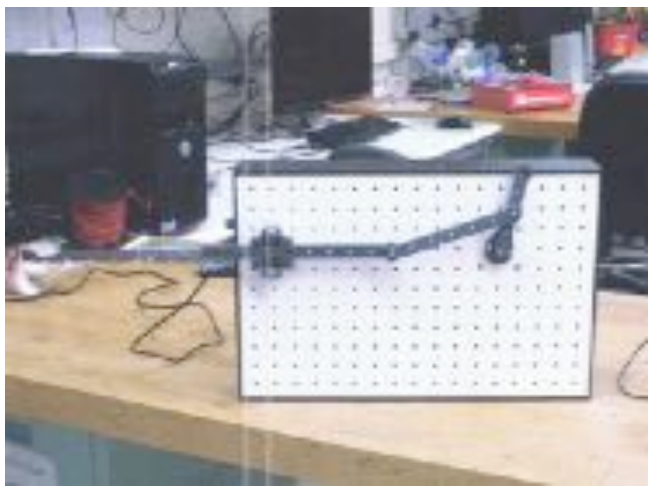

Figure 5, Saw project.

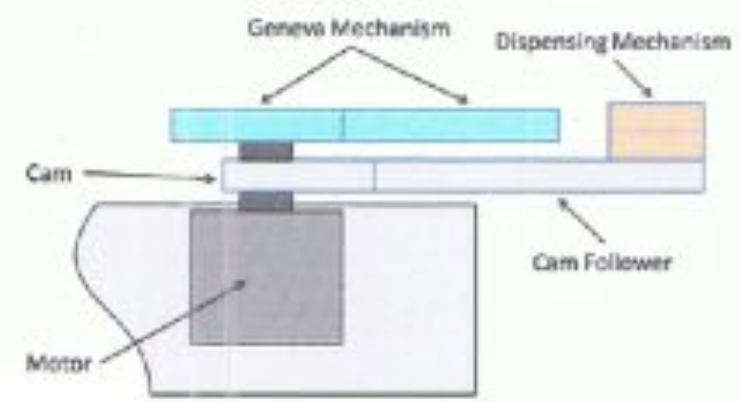

Figure 7(a), Mechanism illustration of the candy dispenser.

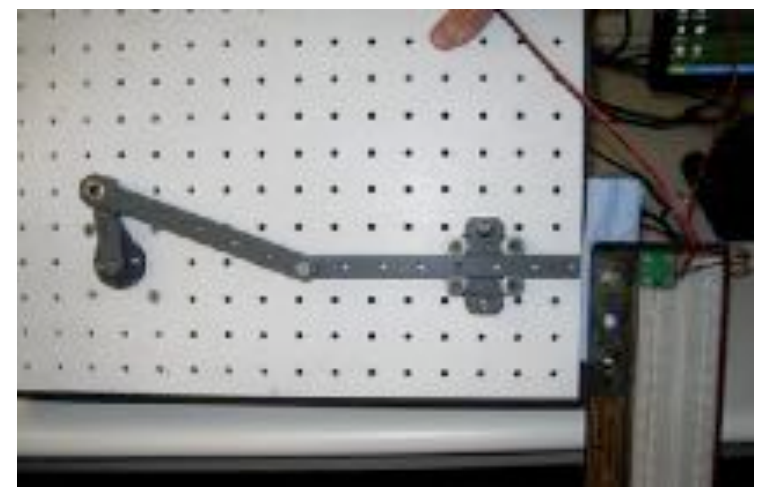

Figure 6, Loco motion with acceleration sensor.

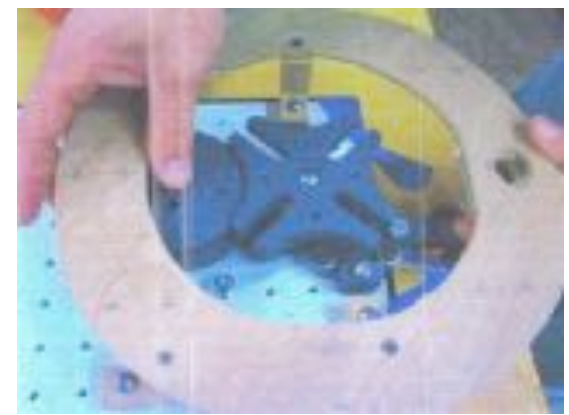

Figure 7(b), Geneva mechanism with wooden base.

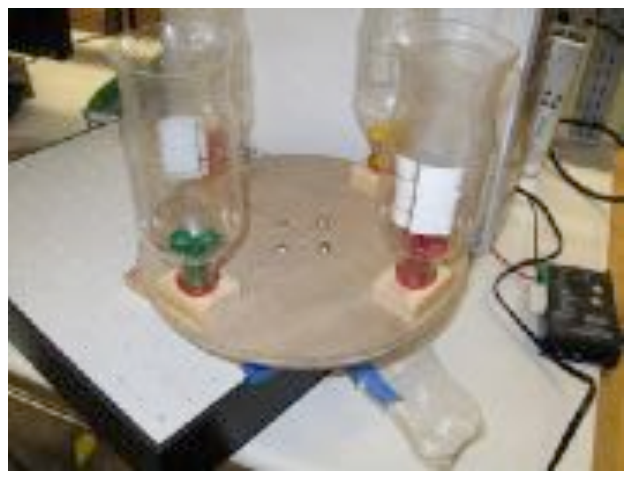

Figure 7(c), Candy dispenser mechanism.

\subsection{Projects Involving Complex Mechanism}

Some students put their vision even further and they want to try more complex mechanisms. Candy dispenser is one of the examples that I want to show. The candy dispenser is capable of storing different kinds of candy. It can dispense the candy at variable speeds depending on what the control signal is set to. This is useful for a college student who likes a little variety in their junk food. This mechanism is a fairly complex machine that utilizes both Geneva mechanism and a cam with an offset follower [4]. There is only one degree of freedom in this mechanism. It 
was designed by first setting up the basic drive mechanism- the kinematics kit. Above the cam was the drive portion of the Geneva mechanism, which was fastened to the cam and hence driven by the motor as shown in Figure 7(a). The Geneva mechanism with the wooden base is then assembled as shown in Figure 7(b). Figure 7(b) also shows how the dispensing mechanism lines up with the hole in the base. This hole is where the candy containers line up with for the $3 / 4$ of the time that they are not in motion. As the cam turn, the dispensing mechanism moves back and forth with the follower. When the cam reaches its maximum displacement, a piece of candy is able to drop in the hole in the dispenser that begins to line up. Underneath the dispensing mechanism there is a stop that keeps the candy from falling all the way out of the machine while the cam is at its maximum displacement. Then as the cam returns to the position of no displacement, the candy is able to drop out of the dispenser and into the serving dish.

The conclusion from the group of students is "we found this to be a fun project to design that ties into the material we have covered in our kinematics class. It would be nice to see our machine implemented into a real life application." In my opinion, this project is really a creative design where a patent can be applied.

Beyond these projects, the walking spider project, obstacle avoiding robot project, nitinol powered walker project, and many others are very creative and interesting. Among them, the obstacle avoiding robot won the third place in the 2010 ASME Student and Mechanism \& Robot Design Competition [5]. All these projects are inspired from engineering problems and in reverse, helped the students understand class materials better.

\section{Conclusion}

A project can help the students understand class materials better. An engineering targeted project can even build more interest for the students to understand the theoretical study in class. At the same time, engineering targeted project can generate creative ideas. Thus we should encourage such project activities for students to combine their class learning with actual engineering problems.

\section{References}

[1] Debao Zhou, http://www.d.umn.edu/ dzhou/ME-3222/Kinematics\%20kits\%20manual.pdf, accessed October 12, 2011.

[2] Sharp Inc. http://www.sharpsma.com/webfm_send/1208, accessed October 12, 2011.

[3] Analog Devices Inc. http://www.analog.com/static/imported-files/eval boards/143456412ADXL321EB 0.pdf, accessed October 12, 2011.

[4] Kenneth Waldron and Gary Kinzel, Kinematics, Dynamics and Design of Machinery, 2nd edition, Wiley \& Sons, 2003, ISBN-13: 978-0471244172.

[5] Jonas Swedberg, "Path Finding Track Vehicle", 2010 ASME Student Mechanism \& Robot Design Competition, Montreal, Quebec, Canada, Aug. 15-18, 2010, http://www.stevens.edu/msrobotics/SMRDC2010/. 